\title{
Measurement of Cohesion in Asteroid Regolith Materials
}

\author{
Julie E. Kleinhenz ${ }^{1}$, James R. Gaier ${ }^{2}$, and Deborah L. Waters ${ }^{3}$ \\ NASA Glenn Research Center, Cleveland, $\mathrm{OH} 44135$ \\ Ralph Harvey ${ }^{4}$, Zoe Zeszut ${ }^{5}$, Brandon Carreno ${ }^{6}$, and Patrick Shober ${ }^{7}$ \\ Case Western Reserve University, Cleveland, $\mathrm{OH} 44107$
}

\begin{abstract}
A study has been initiated to examine cohesive forces in asteroid materials to contribute to a better understanding of low density bodies such as asteroids and Phobos, and assist in exploration missions involving interaction with their surface material. The test specimen used in this study was a lightly weathered CM2 meteorite which is spectroscopically similar to Type $\mathrm{C}$ (carbonaceous) asteroids, and thought to have representative surface chemistry. To account for sample heterogeneity, adhesion forces were measured between the CM2 sample and its five primary mineral phase components. These adhesive forces bound the range of cohesive force that can be expected for the bulk material. All materials were characterized using a variety of optical and spectroscopic methods. Adhesive forces on the order of 50 to $400 \mu \mathrm{N}$ were measured using a torsion balance in an ultrahigh vacuum chamber. The mineral samples exhibited clearly different adhesive strengths in the following hierarchy: Serpentine $>$ Siderite $>$ Bronzite $>$ Olivine $\approx$ Fe-Ni.
\end{abstract}

\section{Nomenclature}

$\begin{array}{ll}\text { AES } & =\text { Auger Electron Spectroscopy } \\ C M 2 & =\text { Carbonaceous type of meteorite: group M, type } 2 \\ D V R T & =\text { Differential Variable Reluctance Transducer } \\ E D S & =\text { Energy Dispersive Spectroscopy } \\ \text { FTIR } & =\text { Fourier Transform Infrared } \\ I S R U & =\text { In-Situ Resource Utilization } \\ \text { QPA } & =\text { Quantitative Phase Analysis } \\ S E M & =\text { Scanning Electron Microscope } \\ U H V & =\text { Ultrahigh Vacuum } \\ W P F & =\text { Whole Pattern Fitting } \\ X R D & =\text { X-ray Diffraction } \\ C & =\text { Cohesion } \\ F & =\text { Adhesive force } \\ f & =\text { Frequency } \\ I & =\text { Moment of Inertia } \\ K & =\text { Spring constant } \\ r & =\text { Length of the lever arm (torsion bar half length) } \\ x & =\text { Displacement } \\ \theta & =\text { Angle of deflection } \\ \sigma & =\text { load normal to the shear plane } \\ T & =\text { Torque }\end{array}$

\footnotetext{
${ }^{1}$ Research Aerospace Engineer, Chemical and Thermal Propulsion Branch, MS 86-8

${ }^{2}$ Research Physicist, Environmental Effects and Coatings Branch, MS 106-1

${ }^{3}$ Materials Research Engineer, Environmental Effects and Coatings Branch, MS 106-1

${ }^{4}$ Professor, Department of Earth, Environmental, and Planetary Sciences, 112 A.W. Smith Bldg.

${ }^{5}$ Graduate Student, Department of Earth, Environmental, and Planetary Sciences, 112 A.W. Smith Bldg.

${ }^{6}$ Graduate, Department of Earth, Environmental, and Planetary Sciences

${ }^{7}$ Undergraduate Student, Department of Earth, Environmental, and Planetary Sciences, 112 A.W. Smith Bldg.
} 


\section{Introduction}

$I^{\mathrm{N}}$ $\mathrm{N}$ the guiding document for NASA's planetary science program, "Vision and Voyages for Planetary Science in the Decade 2013-2022", are found words that stress the importance of studying the primitive bodies of the solar system. "Studies of primitive bodies encompass asteroids, comets, Kuiper belt objects (KBOs), the moons of Mars, and samples-meteorites and interplanetary dust particles (IDPs) — derived from them. These objects provide unique information on the solar system's origin and early history and help interpret observations of debris disks around other stars." So any effort that will elucidate the nature of these primitive bodies will help to unravel important science questions. There is particular interest in asteroids not only for the role they play in the evolution of the solar system, but also as human exploration targets; as sources of resources such as water, fuel, and metals; and for understanding how to mitigate the threat posed by their collision with Earth.

There is increasing evidence that a large fraction of asteroids, and even Mars' moon Phobos, have such low densities $\left(<2 \mathrm{~g} / \mathrm{cm}^{3}\right)$ as to make it unlikely that they conform to the classic image of giant, consolidated "rocks in space”. Many of these also orbit close enough to the sun as to make water a dominant part of their mass highly unlikely. Instead, many of these asteroids are thought to be made up of unconsolidated smaller particles of varying size referred to as "rubble piles". Images of the asteroid Itokawa (Fig. 1) reinforce this hypothesis.

It has been demonstrated that gravitational forces alone are not strong enough to hold together rubble pile asteroids, at least not those that are rapidly spinning. ${ }^{1}$ It has been suggested that van der Waals forces must therefore be responsible for holding them together, but previous work suggests that electrostatic forces, which are orders of magnitude stronger, are far more likely. ${ }^{2}$ Charge build-up is a likely consequence of the interaction of airless bodies with the solar wind plasma, analogous to what has been proposed to occur on the moon. ${ }^{3}$

Adhesion of asteroid regolith presents a risk to the systems and astronauts which will be deployed near the surface of asteroids. For example, Lunar dust posed a wide variety of problems during the Apollo program, severely degrading thermal control surfaces, mechanisms, optical surfaces, seals, fabrics, and perhaps even astronaut health. ${ }^{4}$ Likewise the geotechnical properties of regolith, including adhesion and cohesion, will impact any methods that could be used to acquire and/or process
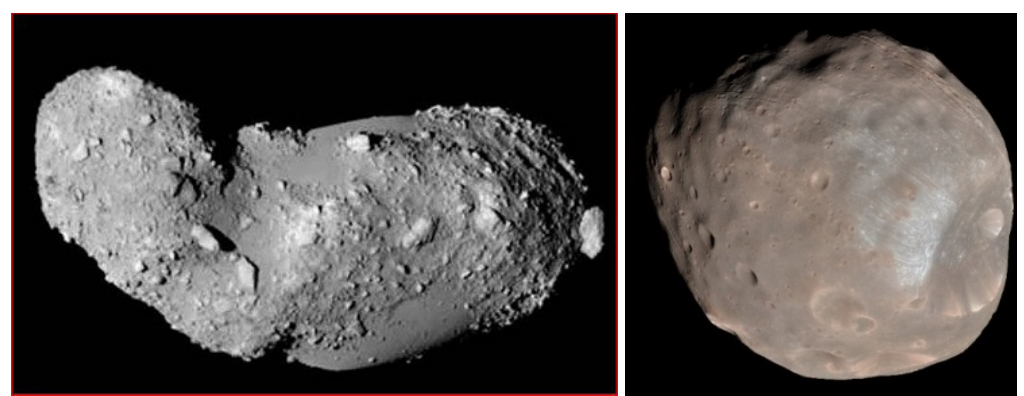

Figure 1. The asteroid Itokawa (left) and the Martian moon Phobos (right) have densities too low to be single, consolidated rocky bodies. They are also too small to be held together primarily by gravity.

regolith; e.g. for scientific sampling/analysis or In-Situ Resource Utilization (ISRU) efforts. Although there has been early theoretical treatment of electrostatically induced dust motion near the surface of an asteroid ${ }^{5}$, to date there have been no laboratory experiments reported to validate the models.

In this study adhesion measurements were made using a torsion balance under ultrahigh vacuum (UHV, $10^{-10}$ Torr or less). The test specimen was a lightly weathered CM2 meteorite obtained from the Antarctic Search for Meteorites program (LON94101), curated by NASA. This meteorite is spectroscopically similar to Type C (carbonaceous) asteroids, and thought to have representative surface chemistry. The meteorite sample was characterized to determine its primary mineral phase components. The adhesion between this CM2 meteorite and terrestrial minerals similar to those found in the meteorite was measured in order to determine the range of cohesion forces that could be expected to hold a Type $\mathrm{C}$ asteroid together.

A distinction is made between regolith cohesion from a geological perspective and cohesive force. Cohesive force, which was the type of measurement done here, is the force required to separate two bodies of the same material, analogous to the adhesion force between unlike materials. Regolith cohesion, $c$, (or cohesive stress) is defined in terms of shear strength, $\tau$, according to $\tau=c+\sigma \tan \varphi$, where $\sigma$ is the load normal to the shear plane, and $\varphi$ is the angle of friction. Cohesion is determined by capillary forces in the presence of fluids (e.g. by humidity), mechanical interlocking, and cohesive and adhesive forces (including electrostatic forces and chemical bonding). Although the ultimate goal is to determine the regolith cohesion in Type $\mathrm{C}$ asteroids, on a microscopic level there will be a substantial adhesive force component, since the regolith grains are themselves heterogeneous in composition. 


\section{Methods}

The first step was to characterize the LON94101 meteorite using energy dispersive spectroscopy (EDS) to determine the primary mineral phases and abundances. This information was used to identify the minerals to use for the adhesion measurements. The mineral samples were then analyzed using x-ray diffraction (XRD) to determine crystallography. An optical surface profilometer was used to inspect surface roughness of all the samples. Prior to adhesion measurements, Auger Electron Spectroscopy was used to verify cleanliness of the samples. While not included in this manuscript, optical and thermal properties of powdered mineral samples were explored with Fourier Transform Infrared (FTIR) and UV-Vis-NIR spectrophotometers. These efforts will feed into a broader study looking at regolith dust transport, thermal and optical degradation similar to what was done for lunar regolith and its simulants in previous work ${ }^{6}$.

\section{A. Characterization of Samples}

A seven segment transect of a $1 \mathrm{~mm}$ thin section of CM2 meteorite LON94101 was examined with a petrographic microscope, using both reflected and transmitted light. Throughout this examination, several images of the sections were taken, to qualitatively show variation in the sections. After this was performed, the section was carbon-coated, and loaded into a JSM-840A scanning electron microscope (SEM). The section was thoroughly photographed for the purpose of creating a mosaic, and a grayscale image of the entire meteorite thin section was constructed. Spatial element abundance maps of the entire mosaic were generated using energy dispersive spectroscopy (EDS). The fraction of each mineral phase was then determined using the Oxford Instruments AZtec software package. Several images in one transect across the section were analyzed and used to determine the relative abundances of mineral phases at higher resolution. This information was used to select the component mineral samples for adhesion testing; Serpentine, Siderite, Olivine, Bronzite, and Fe-Ni.

X-ray Diffraction (XRD) was then used to characterize the crystallography and phase abundances of the adhesion test samples. However, there were insufficient quantities of the CM2 meteorite material and the olivine to perform quantitative XRD analysis on those materials. The serpentine, bronzite and siderite were wet ground in ethanol in a Micronizer (McCone) vibrating mill for 90 minutes, dried overnight at $80^{\circ} \mathrm{C}$. The samples were run in side-loaded holders on a Bruker D8 Advanced diffractometer in Bragg-Brentano mode with a LynxEye linear strip detector using $\mathrm{Cu}$ Ka radiation. Whole pattern fitting (WPF) was performed on the samples using the Jade (MDI) analysis software, which yielded quantitative phase analysis (QPA) and lattice parameters. The Fe-Ni meteorite, which was not ground, sample was hand-polished to remove cold-working. This sample was run on a Bruker D8 Discover diffractometer in point-focus mode with a Vantec500 area detector using Mn Ka radiation. Due to the large grain sizes, QPA could not be performed on the Fe-Ni sample. However, the phases present were identified along with qualitative abundance.

\section{B. Adhesion measurements}

Adhesive force was measured using a torsion balance in an UHV chamber ${ }^{7}$. The torsion balance, shown in Fig. 2, consists of a sample plate (10 mm square) and a displacement sensor mounted on opposite ends of a bar. The bar is suspended horizontally on a taut wire. The sensor consists of a copper block, mounted on the torsion bar, and an UHV compatible Differential Variable Reluctance Transducer (DVRT) which remains fixed. The second sample material is in the form of a pin mounted to an actuated arm with four degrees of freedom. The $\sim 3 \mathrm{~mm}$ diameter pin has a rounded end to control surface contact

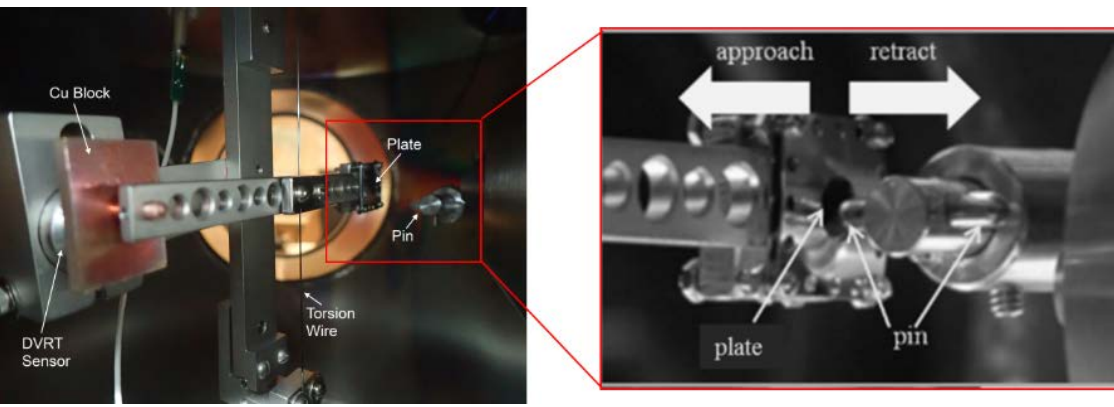

Figure 2. The torsion balance in the UHV chamber which used for adhesive force measurement.

area (Hertzian) when the pin is brought into contact with the plate. To initiate the adhesive force measurement, the pin is loaded against the plate causing the bar to twist around the wire. The torsion spring force of the wire resists this applied force. The two forces are in equilibrium, so the angle of bar deflection, along with the spring constant of the wire and bar length, can be used to calculate the applied force. Adhesive force is measured by slowly retracting the 
pin away from the plate. If adhesion exists, the bar deflects in the direction of the pin. The two forces are in equilibrium just before the spring force of the wire overcomes the adhesion force, and the plate pulls back to its rest position.

The governing equation to calculate the adhesion force is Hooke's Law for an angular force:

$$
\mathrm{T}=\mathrm{K} \theta
$$

where the terms are torque $(T)$, spring constant $(K)$, and the angle of deflection $(\theta)$. The spring constant can be obtained from the natural resonant frequency of the balance, provided that damping is small (which it is in this case) from:

$$
f=1 / 2 \pi \sqrt{ }(\mathrm{K} / \mathrm{I})
$$

where (f) is the frequency and (I) is the moment of inertia, both of which can be measured directly. The angle of displacement $(\theta)$ is obtained by the displacement $(\mathrm{x})$ measured by the DVRT operation using

$$
\tan \theta=\mathrm{x} / \mathrm{r} \text {. }
$$

The adhesion force $(\mathrm{F})$ was then determined from the torque and the length of the lever arm (r).

$$
\mathrm{F}=\mathrm{T} / \mathrm{r} \text {. }
$$

The output signal of the DVRT sensor was collected in Labview ${ }^{\circledR}$ at $200 \mathrm{~Hz}$ and processed in IGOR Pro ${ }^{\circledR}$. In its current configuration the sensor can detect forces between $5 \mu \mathrm{N}$ (noise level) to $5000 \mu \mathrm{N}$. For the forces measured in this study (on the order of $200 \mu \mathrm{N}$ ) the total uncertainty ${ }^{8}$ of the force measurement is approximately $35 \mu \mathrm{N}$. This includes uncertainties in the measurements, calibration factors, and sensor noise.

The torsion balance was mounted inside an UHV chamber shown in Fig 3. The chamber is equipped with a combination of sorption, sublimation, and ion pumps (oil-free) to reach a pressure on the order of $1 \times 10^{-10}$ Torr. The entire system rests on a vibration isolation table to minimize external vibrations that could interfere with the sensitive torsion balance. An Ion Bombardment Gun and an Auger Electron Spectrometer (AES) facilitate cleaning of the samples under vacuum. Each day prior to testing, the samples were examined with the AES, then sputtered in an argon environment for approximately 20 minutes. This is repeated until the AES reflects a significant reduction in the amount of surface contaminates (e.g. carbon). Given that the plate is a carbonaceous meteorite, cleaning focused on the pin samples in this study. For each pin type approximately 150 adhesion tests were performed. The ion pump remained active during all adhesion runs to maintain the UHV. The pin was loaded against the plate with forces on the order of $1000 \mu \mathrm{N}$ and remained in contact for approximately 30 seconds. Previous studies ${ }^{2}$, and initial check out tests, have shown that load force and time do not have a significant effect on the adhesive force using this method. The pin position relative to the plate was varied to account for heterogeneities in the CM2 meteorite sample and to periodically present a fresh pin contact surface. This rastering typically consisted of 18 locations over the plate surface. The angle

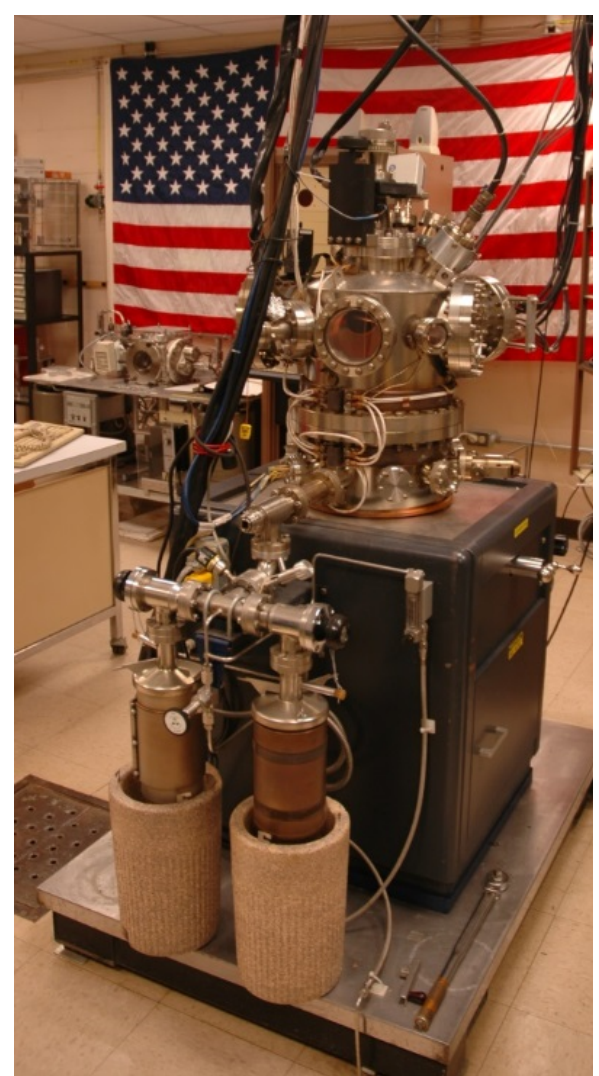

Figure 3. The UHV chamber. of the pin was also varied to account for any irregularities in the pin samples. Three pin angles were used; perpendicular to the plate ("straight") and angles of approximately $\pm 45^{\circ}$ (designated as "up" and "down"). Electrostatic charging was induced for some tests by impacting the pin against the plate. This was done by bringing the pin and plate close together and then tapping on the pin mechanism, and was designated as a 'hammer strike'. The raw data was imported into IGOR Pro ${ }^{\circledR}$. for analysis. Due to the large number of runs (775 total) data processing was automated using custom IGOR Pro ${ }^{\circledR}$ procedures. 


\section{Results and Discussion}

The results in the following sections review the characterization of both the CM2 meteorite and pin materials as well as the preliminary results from the adhesion tests. The analysis of this data is ongoing, with a more comprehensive publication to follow.

\section{A. Sample material characterization}

Figure 4 is a photomicrograph that shows a slice from the surface of the CM2 meteorite. It is color coded to show the concentrations of $\mathrm{Ca}$, $\mathrm{Fe}$, $\mathrm{Mg}$, and S. Analysis of maps like this led to a quantification of the mineral types and abundances found in this sample. The results are shown in the table beside the figure. This image also makes clear the heterogeneity of the sample. Note that the large circular structure is a chondrule (Olivine / Pyroxene). This adds some complication to cohesive force testing since there is no guarantee that like materials are in contact. For example, if the pin and plate were both made from CM2 material, it would be possible for a phyllosilicate dominant region to make contact with the chondrule, depending on the orietation of the two samples. This is why the pin samples were made of the

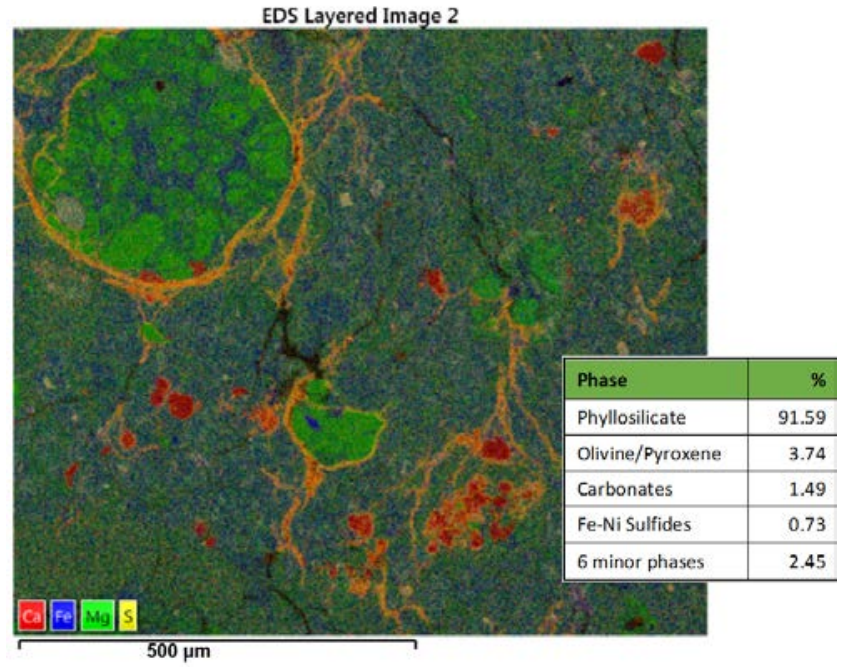

Figure 4. The photomicrograph used to determine the primary mineral phases of the CM2 meteorite. component mineral phases. By moving across the CM2 sample during adhesion testing for each pin these variations would balance out to give an approximation for bulk cohesion.

Over $90 \%$ of the CM2 meteorite is made up of a phyllosilicate mineral. A close match to the mineralogy is the terrestrial mineral serpentine. Therefore, the most characteristic cohesion test would be the serpentine/CM2 pair. The lesser component phases were also used: olivine, pyroxene (bronzite), iron carbonate (siderite), and iron/nickel (Fe$\mathrm{Ni}$ ) metal. The minor phases such as boron, Ca-Fe sulfides, Ca-Fe oxides, gypsum, $\mathrm{Cr}-\mathrm{Fe}$, and Al-silicates were not included because they were compositionally insignificant for this study. Therefore pins were manufactured from serpentine, olivine, bronzite, siderite and Fe-Ni.

All the pins were cut from the source material using a diamond saw and shaped using standard machining equipment. Prior to testing they were polished using diamond paste and thoroughly cleaned with ethyl alcohol. The CM2 meteorite plate and pin samples are shown below in Figure 5. The majority of the source materials were provided by the Environmental and Planetary Sciences Department at Case Western Reserve University. This includes siderite,
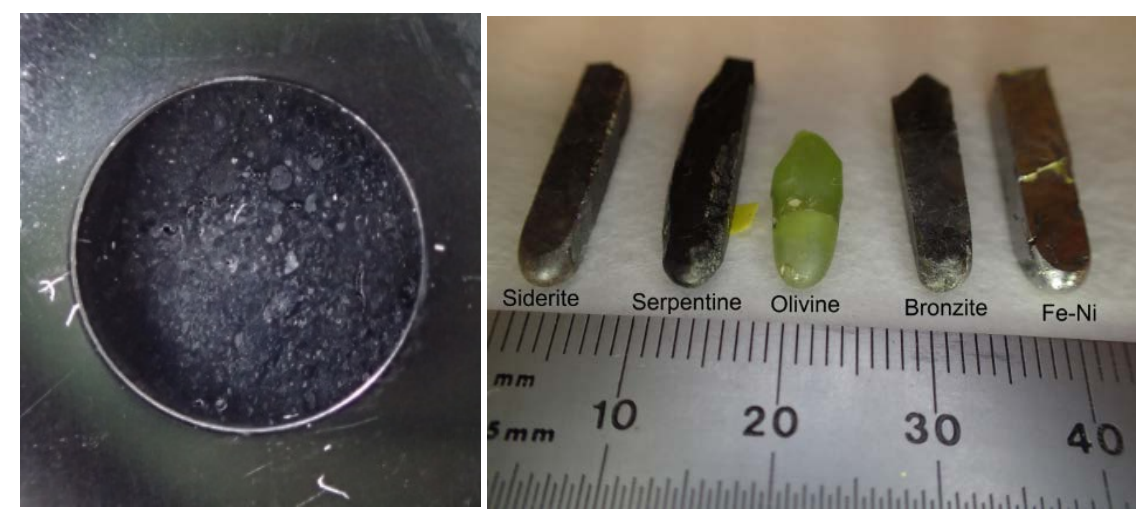

Figure 5. The samples used in the cohesion testing. At left is the CM2 meteorite sample mounted in the plate holder with an orifice of about $0.6 \mathrm{~cm}$, and at right are the different pin samples used. bronzite (collected Jackson County, NC), gem-quality Olivine, and Fe-Ni (from the Gibeon meteorite). The serpentine was sourced from Wards Scientific. Two serpentine pins and two olivine pins were manufactured and tested due to alterations in the machining process. However, there was no apparent difference in the adhesive force results.

A VEECO optical profilometer was used to image the surfaces of the pin tips both before and after adhesion runs in the chamber. The pre-chamber scans were done in order to inspect the surfaces in more detail and to try to identify any major surficial irregularities or defects that could have an effect on adhesion force. In some cases, additional polishing was performed to smooth out any excessive surface roughness. Because the pins were fabricated 
from natural (often brittle) materials with inherent crystal structures, they could not be made perfectly smooth. The olivine pin pictured in Fig. 6 is one example of the profilometry results. The small divot in the top of the pin can be seen, and its depth measured, using this method. Pins and plates were also scanned with the profilometer after they had completed adhesion tests. These new images were compared with the pre-test images to verify there was no transfer of material between the pin and the plate during testing. Thus far there have been no observable changes in the surface profiles so reuse of both the pin and plate samples remains possible.

\section{B. X-Ray Diffraction Characterization of Powders}

$\mathrm{XRD}$ is used here primarily for phase identification of crystalline material. Average bulk composition can also be determined if the material is ground up finely, but not too fine to obscure the crystallinity. Table 1 shows the crystallography of each of the materials. For each of the powdered samples the weight percent with error in parenthesis is shown in the last column of the table. These error values do not represent the full possible error, but rather give a standard statistical error regarding the fit of the model parameters to the data. For the Fe-Ni pin, only relative abundance could be determined because the material was not ground. After hand-polishing this sample to remove the cold working layer, SEM was performed to gather chemistry data on local features via EDS.
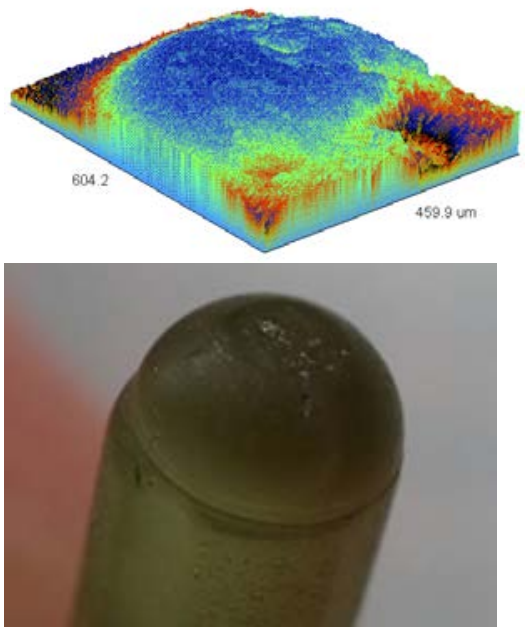

Figure 6. An example of the profilometry data (top) and its corresponding visual image of the Olivine pin (bottom).

\begin{tabular}{|c|c|c|c|c|c|}
\hline Sample & Phase & $\begin{array}{c}\text { Chemical Formula } \\
\text { (nominal) }\end{array}$ & $\begin{array}{l}\text { Crystal } \\
\text { System }\end{array}$ & Space Group & $\begin{array}{l}\text { wt \%* or } \\
\text { Relative } \\
\text { Abundance } \\
\text { (error) }\end{array}$ \\
\hline serpentine \#2 & $\begin{array}{l}\text { chrysotile } \\
\text { antigorite } \\
\text { chlorite } \\
\text { magnetite }\end{array}$ & $\begin{array}{l}\text { Mg3Si2O5(OH)4 } \\
\text { Mg3Si2O5(OH)4 } \\
\text { (Mg,F3)6(Si,Al)4O10(OH)8 } \\
\text { Fe3O4 }\end{array}$ & $\begin{array}{l}\text { monoclinic } \\
\text { monoclinic } \\
\text { anorthic } \\
\text { cubic }\end{array}$ & $\begin{array}{c}\mathrm{C} 2 / \mathrm{m}(12) \\
\operatorname{Pm}(6) \\
\mathrm{C} 1(1) \\
\mathrm{Fd}-3 \mathrm{~m}(227)\end{array}$ & $\begin{array}{l}64.1(8) \\
22.4(4) \\
11.2(3) \\
2.4(1)\end{array}$ \\
\hline serpentine \#3 & $\begin{array}{c}\text { antigorite } \\
\text { magnesite } \\
\text { magnetite } \\
\text { periclase }\end{array}$ & $\begin{array}{l}\mathrm{Mg} 3 \mathrm{Si} 2 \mathrm{O} 5(\mathrm{OH}) 4 \\
\mathrm{Mg}(\mathrm{CO} 3) \\
\mathrm{Fe} 3 \mathrm{O} 4 \\
(\mathrm{Mg}, \mathrm{Fe}) \mathrm{O}\end{array}$ & $\begin{array}{l}\text { monoclinic } \\
\text { hexagonal } \\
\text { cubic } \\
\text { cubic }\end{array}$ & $\begin{array}{c}\text { Pm (6) } \\
\text { R-3c (167) } \\
\text { Fd-3m (227) } \\
\text { Fm-3m (225) }\end{array}$ & $\begin{array}{l}85(1) \\
11.7(4) \\
2.3(2) \\
0.7(1)\end{array}$ \\
\hline bronzite & $\begin{array}{l}\text { anthophyllite } \\
\text { enstatite } \\
\text { clinochlore }\end{array}$ & $\begin{array}{l}\text { Mg7Si8O22(OH)2 } \\
\text { MgSiO3 } \\
(\mathrm{Mg}, \mathrm{Al}) 6(\mathrm{Si}, \mathrm{Al}) 4 \mathrm{O} 10(\mathrm{OH}) 8\end{array}$ & $\begin{array}{l}\text { orthorhombic } \\
\text { orthrhombic } \\
\text { monoclinic }\end{array}$ & $\begin{array}{l}\text { Pnma (62) } \\
\text { Pbca (61) } \\
\text { C2/c (15) }\end{array}$ & $\begin{array}{c}54.8(6) \\
44.3(6) \\
0.9(1)\end{array}$ \\
\hline siderite & $\begin{array}{c}\text { siderite } \\
\text { low quartz }\end{array}$ & $\begin{array}{l}\mathrm{Fe}(\mathrm{CO} 3) \\
\mathrm{SiO} 2\end{array}$ & $\begin{array}{l}\text { hexagonal } \\
\text { hexagonal }\end{array}$ & $\begin{array}{l}\text { R-3c (167) } \\
\text { P3221 (154) }\end{array}$ & $\begin{array}{l}97(1) \\
3.0(2)\end{array}$ \\
\hline Fe-Ni pin & $\begin{array}{c}\text { kamacite } \\
\text { cohenite } \\
\text { taenite } \\
\text { schreibersite }\end{array}$ & $\begin{array}{l}(\mathrm{Fe}, \mathrm{Ni}) \\
\mathrm{Fe} 3 \mathrm{C} \\
(\mathrm{Fe}, \mathrm{Ni}) \\
(\mathrm{Fe}, \mathrm{Ni}) 3 \mathrm{P}\end{array}$ & $\begin{array}{c}\text { cubic } \\
\text { orthorhombic } \\
\text { cubic } \\
\text { tetragonal }\end{array}$ & $\begin{array}{l}\text { Im-3m (229) } \\
\text { Pmna (62) } \\
\text { Fm-3m (225) } \\
\text { I-4 (82) }\end{array}$ & $\begin{array}{l}\text { major } \\
\text { major } \\
\text { minor } \\
\text { minor }\end{array}$ \\
\hline
\end{tabular}

${ }^{*}$ For powdered samples the number in this column refers to weight percent (wt\%)

${ }^{*}$ For solid samples (Fe-Ni) the number in this column refers to relative abundance 


\section{Adhesion Measurements}

A typical plot of one adhesion run is shown in Fig. 7. Force is plotted as a function of elapsed time. Negative force represents loading of the pin against the plate, while positive force is pull-off (adhesive) force. In the first $20 \mathrm{~s}$ the pin is pushed (loaded) against the plate with a force of $1600 \mu \mathrm{N}$. The pin is then slowly retracted away from the plate at a rate of approximately $9 \mu \mathrm{m} / \mathrm{s}$, which corresponds to a load reduction of about $70 \mu \mathrm{N} / \mathrm{s}$. At $45 \mathrm{~s}$ the plate is pulled past its equilibrium point until a force of about $200 \mu \mathrm{N}$ exerted by the torsion wire pries the plate and pin apart. This force is the adhesive force. The torsion balance oscillates freely between $45 \mathrm{~s}$ and $75 \mathrm{~s}$ where there is no contact between the pin and plate. At $75 \mathrm{~s}$ the pin is moved back towards the plate for the next run. As this occurs, the plate lunges towards the plate, presumably due to electrostatic attraction caused by contact charging. In this report the term "attraction" will be reserved for this interaction rather than for adhesive or cohesive forces which are also attractive. After $75 \mathrm{~s}$, the pin continues to move against the plate in preparation for the next run.

Figure 8 shows the data summary from all the Serpentine runs as an example

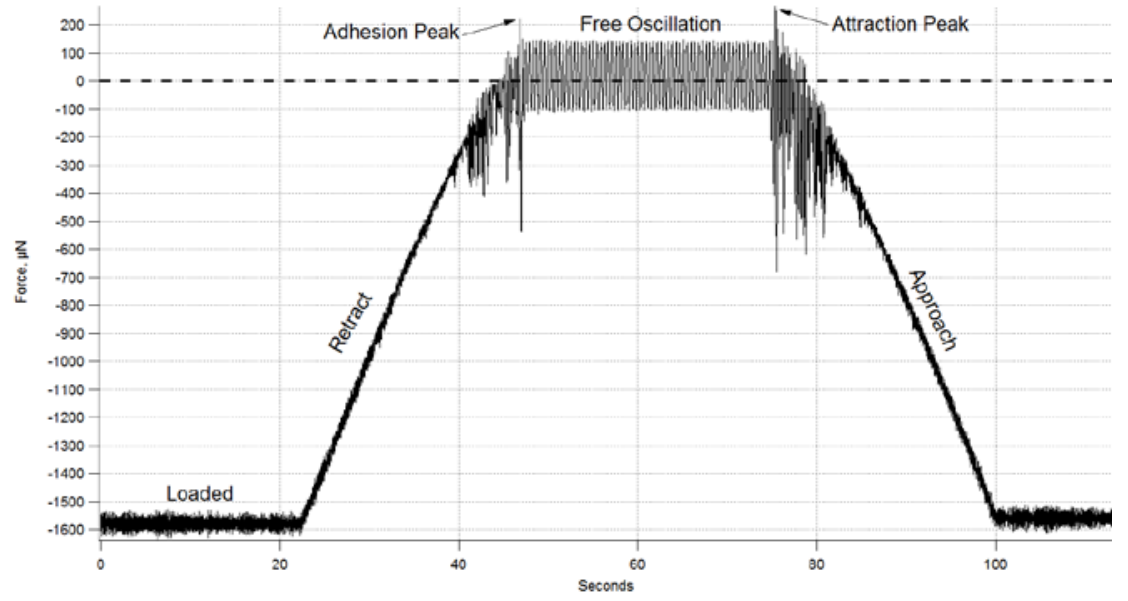

Figure 7. An example of a single adhesion run.

of the results. The run number is plotted on the x-axis with measured force on the left axis. Adhesion peaks are represented by downward triangles while attraction peaks are upward triangles. The cross symbols represent the peak of the free oscillation. Run numbers with no symbols indicate that there was no defined peak for either adhesion or attraction. At the point of pull-off or approach the force peak had to be $5 \mu \mathrm{N}$ greater than the free oscillation peak for adhesion/attraction to be positively identified. This was a way to define clear peaks in the data set for this preliminary analysis. However, as data analysis continues this threshold will be revisited and refined. The magnitude of the free oscillation peak may also be an indicator of relevant forces. The vertical red bars in Fig. 8 also indicates where hammer strikes were used to induce electrostatic charge. This charge will linger for some as-yet-undefined time so the gray bars, corresponding to the right axis, are an indicator of this. The thick black line represent the start of a new test day, where the samples would be re-cleaned.

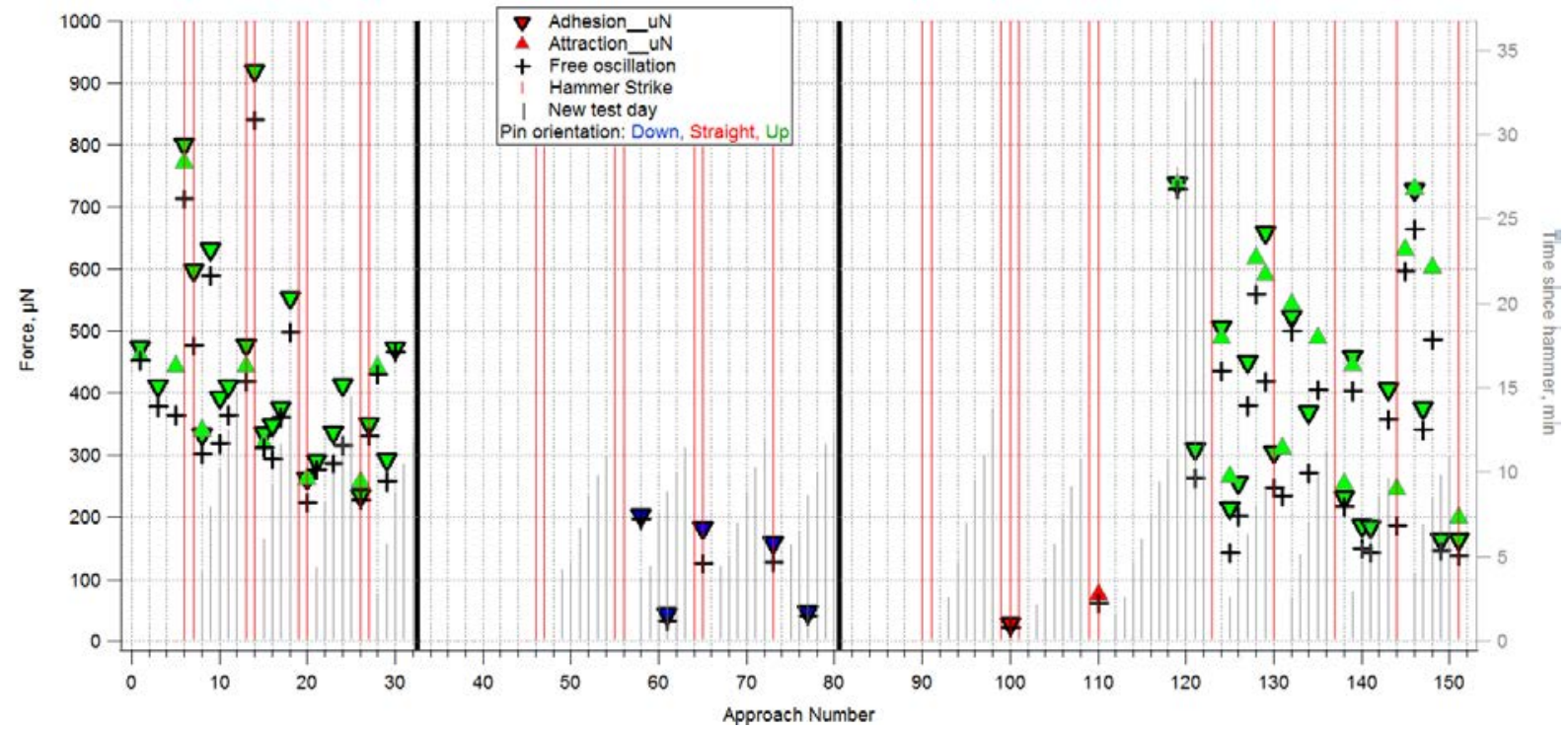

Figure 8. The summary of all the adhesion tests using Serpentine pins. 
Given that both the hammer strikes and the ion-cleaning at the start of each day are potential sources of electrostatic charge, it is challenging to make a clear distinction of adhesion dominated by van der Waals forces. However there are two potential indicators. First, attraction peaks must be caused by electrostatics because van der Waals forces do not act over such long distances. Second, adhesive force that immediately follows a hammer strike is likely due to electrostatic forces. Therefore, Van der Waals forces can only be definitive when there is no attractive peak immediately following a pull off (unlike the example of Fig.7) and after sufficient time has dissipated electrostatic charge from either an ion cleaning or hammer strike. The histogram in Fig. 9 is the first attempt to define

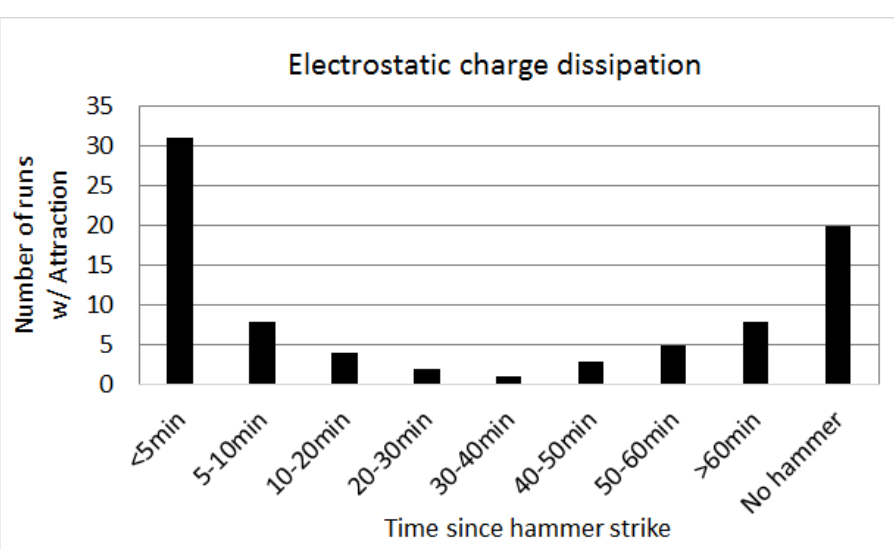

Figure 9. A histogram correlating attraction force to electrostatic charging events. The "no hammer" data was taken at least 1 hour after ion cleaning of the surfaces. electrostatic charge dissipation time. The data in the 'no hammer' category were only exposed to ion cleaning. For all tests, at least one hour passed between ion cleaning and the first test run. The rest of the data mark the time since the last hammer strike and suggest that electrostatic charge significantly dissipates after only 5 minutes. However this does not reflect how many times the pin and plate touch, which could dissipate the charge quicker. Likewise, the metal sample holders for both pin and plate are suspended on actuated chamber feedthroughs whose grounding paths have yet to be addressed. It also is unclear why the number of attraction occurrences begins to rise again after 30 minutes. The interpretation of these data is ongoing.

A total of 775 adhesion runs were performed between the CM2 meteorite and five of its mineral phase components. An overview of the results are shown in Table 2. Adhesive force was only observed in about 12 percent of all tests, and attraction in 11 percent. Of all the measured adhesive forces, potentially $8 \%$ can be attributed to van der Waals forces. However, there are still a number of open questions that will need to be addressed on further analysis. For example, the majority of runs show no adhesive forces at all. In Fig. 8, there seems to be some dependence on pin orientation, where adhesion is more prevalent with pins tilted upward. The same dependency, with the same pin orientation, was seen in the Siderite results. Profilometery of these pins did not suggest any irregularities in the pin geometries that would account for this. Also, some runs that showed attraction peaks did not show adhesion peaks which seems odd if an electrostatic charge was truly present. Additionally, one would expect the Van der Waals forces would be lower than those attributed to electrostatic, but the data shows no clear trend to this effect.

Nevertheless, several qualitative observations can be drawn from the preliminary results. From Table 2 it is evident that the different minerals exhibit different adhesive strengths. Serpentine, which is the dominant mineral phase of the CM2 meteorite, and thus the most representative of cohesion, clearly exhibits the strongest adhesive force. Serpentine is a phyllosilicate (or "sheet silicate"), and this fibrous and platy structure is a likely factor for its greater adhesion. Siderite exhibits the next strongest adhesive strength, though the probability of occurrence is nearly half that of serpentine. Bronzite follows with even fewer clear instances of adhesion. Olivine and Fe-Ni are similar with the lowest adhesive potential of the samples tested.

\begin{tabular}{|l|r|r|r|r|r|r|}
\hline & Serpentine & Siderite & Bronzite & Olivine & FeNi & All Tests \\
\hline Total number runs & 154 & 144 & 157 & 184 & 136 & 775 \\
\hline Run with Adhesion & $30.5 \%$ & $14.6 \%$ & $10.8 \%$ & $3.8 \%$ & $2.9 \%$ & $12.4 \%$ \\
\hline Runs with Attraction & $15.6 \%$ & $14.6 \%$ & $11.5 \%$ & $4.3 \%$ & $8.1 \%$ & $10.6 \%$ \\
\hline Runs w Adhesion only & $20.8 \%$ & $10.4 \%$ & $6.4 \%$ & $3.8 \%$ & $2.2 \%$ & $8.6 \%$ \\
\hline Runs with Attraction only & $5.8 \%$ & $10.4 \%$ & $7.0 \%$ & $4.3 \%$ & $7.4 \%$ & $6.8 \%$ \\
\hline Runs with adhesion \& attraction & $9.7 \%$ & $4.2 \%$ & $4.5 \%$ & $1.1 \%$ & $0.7 \%$ & $4.0 \%$ \\
\hline
\end{tabular}

Table 2. A summary of all the test results. 


\section{Conclusion}

A study has been initiated to examine cohesive forces in asteroid materials. As the first effort to directly measure these forces, this knowledge will not only contribute to a better understanding of low density bodies such as asteroids and moons like Phobos but also assist in exploration endeavors involving interaction with their surface material. Knowledge of the properties of dust and regolith, such as cohesion, will impact seals, mechanisms, and possibly even astronaut health for any mission that may visit such bodies.

A lightly weathered CM2 meteorite was selected as the most representative of common carbonaceous asteroids. To account for sample heterogeneity, adhesive forces were measured between the CM2 sample and five of its primary mineral phase components. These adhesive forces bound the range of cohesive forces that can be expected for the bulk material. Characterization of the sample using EDS revealed its primary mineral phases and representative terrestrial samples were selected to use in the adhesion tests. The mineral samples were characterized to determine crystallography and mineral abundance using XRD. Surface profilometry was performed to characterize roughness of the samples used in the adhesion tests. Additional studies examining thermal and optical properties of all materials will be offered in subsequent publications.

Several qualitative conclusions can be drawn from the preliminary adhesive force results. First, adhesive and attractive forces on the order of 50 to $400 \mu \mathrm{N}$ were detected between a CM2 meteorite and its component minerals. Second, van der Waals forces can be distinguished from electrostatic forces based on the conditions surrounding the occurrence of adhesion. Finally, the different minerals exhibit clearly different adhesive strengths. Serpentine clearly exhibits the strongest adhesive forces, with the rest of the minerals exhibiting forces in the following hierarchy: Serpentine $>$ Siderite $>$ Bronzite $>$ Olivine $\approx$ Fe-Ni.

\section{Acknowledgments}

This work was supported by the NASA Science Innovation Fund program under the Office of the Chief Scientist. Rick Rogers, NASA Glenn Research Center, performed the X-Ray Diffraction experiment and analysis, and contributed substantially to this work. The machining work for the pin samples was performed by Frank Lam.

\section{References}

${ }^{1}$ B. Rozitis, E. MacLennan, J.P. Emery, “Cohesive forces prevent the rotational breakup of rubble-pile asteroid (29075) 1950 DA”, Nature 13632 (2014).

${ }^{2}$ S. Berkebile and J.R. Gaier, "Adhesion in a Vacuum Environment and its Implications for Dust Mitigation Techniques on Airless Bodies". International Conference on Environmental Systems 2012, AIAA-2012-3465.

${ }^{3}$ D.R. Criswell, "Lunar Dust Motion” Geochemica et Cosmochemica Acta, Vol 3 (1972) 2671-80.

${ }^{4}$ J.R. Gaier, "The Effect of Lunar Dust on EVA Systems During the Apollo Missions", NASA Technical Memorandum, NASA/TM - 2005 - 213610 - REV 1, (2007).

${ }^{5}$ N.D. Borisov and A.V. Zakharov, "Electrostatic Charging and Motion of Dust near the Surface of an Asteroid”, Solar System Research, Vol 48, No. 1 (2014) 24-34.

${ }^{6}$ J.R. Gaier, M.C. Hicks, and R.M. Misconin, "Studies of Simulated Lunar Dust on the Properties of Thermal Control Surfaces" Journal of Spacecraft and Rockets Vol 50 (2013) pp 848-852.

${ }^{7}$ Miyoshi, K., Chikayoshi, M., and Ryuchi, M., “Development of a Torsion Balance for Adhesion Measurements”, NASA TM100799, 1988.

${ }^{8}$ ISO. “Guide to the expression of Experimental Uncertainty”, ANSI/NCSL Z540-2-1997

American Institute of Aeronautics and Astronautics 